\title{
To err is autonomic: Error-related brain potentials, ANS activity, and post-error compensatory behavior
}

\author{
GREG HAJCAK, NICOLE MCDONALD, AND ROBERT F. SIMONS \\ Department of Psychology, University of Delaware, Newark, Delaware, USA
}

\begin{abstract}
A two-component event-related brain potential consisting of an error-related negativity (ERN/Ne) and positivity (Pe) has been associated with response monitoring and error detection. Both the ERN and Pe have been source-localized to the anterior cingulate cortex (ACC) - a frontal structure implicated in both cognitive and affective processing, as well as autonomic nervous system (ANS) modulation. The current study sought to examine the relationships among the ERN, the Pe, two autonomic measures, and behavior. Electroencephalogram (EEG), heart rate (HR), and skin conductance (SC) were recorded while subjects performed a two-choice reaction-time task. In addition to the characteristic ERN-Pe complex, errors were associated with larger SCRs and greater HR deceleration. The ERN correlated with the number of errors, but was unrelated to ANS activity and compensatory behavior. Pe, on the other hand, was correlated significantly with SCR, and both SCR and Pe were significantly correlated with post-error slowing.
\end{abstract}

Descriptors: Error-related negativity (ERN), Event-related brain potential (ERP), Error processing, Heart rate (HR), Skin conductance response (SCR)

The error-related negativity (ERN/Ne) and subsequent error positivity $(\mathrm{Pe})$ is a two-component event-related brain potential (ERP) complex that has been associated with monitoring actions and detecting errors (Gehring, Coles, Meyer, \& Donchin, 1990; Gehring \& Fencsik, 2001; Hohnsbein, Falkenstein, \& Hoorman, 1989). In speeded reaction-time tasks, the ERN is observed as a negative deflection at midline fronto-central recording sites $(\mathrm{Fz}$, $\mathrm{FCz}, \mathrm{Cz}$ ) that begins when an incorrect response is initiated and peaks approximately 50-150 ms later (Dikman \& Allen, 2000; Falkenstein, Hoorman, Christ, \& Hohnsbein, 2000; Gehring, Goss, Coles, Meyer, \& Donchin, 1993; Luu, Collins, \& Tucker, 2000; Nieuwenhuis, Ridderinkhof, Blom, Band, \& Kok, 2001; Scheffers \& Coles, 2000). The Pe has a slightly more posterior scalp distribution, follows the ERN, and peaks approximately 200-400 ms after a performance error occurs (Falkenstein et al., 2000; Hohnsbein et al., 1989; Nieuwenhuis et al., 2001).

Typically, the ERN is discussed in terms of the activity of a general action-monitoring system that operates across various stimulus and response modalities. Specifically, the ERN has been found with both visual and auditory stimuli, and with hand, finger, foot, and eye responses (Bernstein, Scheffers, \& Coles, 1995; Holroyd, Dien, \& Coles, 1998; Van't Ent \& Apkarian, 1999). The ERN is found for errors of commission as well as

Portions of this article were presented at the 42nd annual meeting of the Society for Psychophysiological Research, Washington, DC, October 2002.

Address reprint requests to: Robert F. Simons, Ph.D., Department of Psychology, University of Delaware, Newark, DE 19716, USA. E-mail: rsimons@udel.edu. errors of omission (Falkenstein et al., 2000), and has been shown by Miltner, Braun, and Coles (1997) to occur following an external feedback stimulus indicating an erroneous response when endogenous error signals are unavailable (i.e., when subjects are unsure that they have committed an error; for similar work on the feedback ERN, see Gehring \& Willoughby, 2002; Luu, Tucker, Derryberry, Reed, \& Poulsen, 2003).

There is good evidence that affective and motivational factors can also modulate ERN magnitude. When motivated to make accurate rather than speedy responses, the ERN is larger; likewise, larger ERNs occur when subjects are more certain that they have made an error (Falkenstein et al., 2000; Gehring et al., 1993). Similarly, emotion-related individual difference variables are consistently related to ERN magnitude. Gehring, Himle, and Nisenson (2000), for example, report that the ERN is significantly larger in patients with Obsessive-Compulsive Disorder (OCD) than in normal controls and that symptom severity is positively correlated with ERN magnitude (for similar results with OCD patients, see Johannes et al., 2001). In a related study, Hajcak and Simons (2002) found that the ERN was significantly larger on both error and correct trials in college undergraduates with obsessive-compulsive (OC) characteristics than it was in low-OC control subjects. More recently Hajcak, McDonald, and Simons (in press) found that this enhanced ERN also characterized subjects who were excessive worriers, but not subjects who report only specific phobias.

In contrast to high-anxiety subjects, Dikman and Allen (2000) found that the ERN was selectively reduced in subjects who scored low on measures of socialization - a possible analog of psychopathy. Interestingly, this blunting of the ERN only 
occurred under conditions of punishment - the same subjects had ERNs that were comparable to controls under reward conditions. Last, differences in ERN amplitude have been related to measures of negative affect and negative emotionality more generally (Luu, Collins, et al., 2000). In fact, the ERN has been described by Luu, Fleisch, and Tucker (2000) as a neural signal related to "evaluative self-monitoring along an affective dimension" (p. 469).

Despite the large number of experimental manipulations that have related ERN amplitude differences to motivational and affective variables, far less is known about the relationship between the ERN and behavioral data. For instance, Rabbit (1981) reports that when subjects commit errors in speeded reaction-time tasks, post-error trials are characterized by unusually long reaction times (RT). Presumably, this RT slowdown is a compensatory action taken to increase the likelihood of correct responses on trials subsequent to errors. In this way, post-error RT slowing might serve as a behavioral measure of response monitoring-but the ERN does not consistently relate to this measure (Gehring \& Fencsik, 2001; Gehring \& Knight, 2000; Scheffers, Humphrey, Stanny, Kramer, \& Coles, 1999).

Two recent ERN studies provide further evidence that the ERN and post-error RT slowing are unrelated. Specifically, Nieuwenhuis et al. (2001) measured ERPs while subjects performed an antisaccade task. In their task, subjects would occasionally make errors yet report making the correct response. In terms of these "unconscious" errors, Nieuwenhuis et al. found that subjects did, in fact, generate an ERN; however, these errors were not associated with the post-error RT slowing found on conscious error trials. Likewise, Mathalon et al. (2002) recorded the ERN from schizophrenic and control subjects. Patients with schizophrenia had both smaller ERNs on error trials and larger ERN-like responses on correct trials than the control subjects. Despite these ERN differences between the patients and controls, both groups had comparable post-error RT slowing.

Although far less elaborated in the ERP literature, the error positivity $(\mathrm{Pe})$ also appears to be associated with response monitoring processes. Falkenstein et al. (2000) proposed that the Pe may be related to additional processing that occurs after error detection, such as conscious error recognition. The recent Nieuwenhuis et al. (2001) study has supported this hypothesis by demonstrating a relationship between $\mathrm{Pe}$ and the subjective awareness of an error. When subjects made unconscious errors in their antisaccade task, the Pe was substantially reduced. Interestingly, in the Mathalon et al. (2002) ERN study with patients with schizophrenia, error trials yielded a normal Pe in both schizophrenic and control subjects, and in both the Mathalon et al. and Nieuwenhuis et al. studies, only the trials with an observable Pe were associated with post-error RT slowing. This would suggest that behavioral measures associated with response monitoring may be more related to the Pe than the ERN.

In one of the earliest ERP studies, Gehring et al. (1993) proposed that the neural source of the ERN was the anterior cingulate cortex (ACC). Since then, numerous studies that utilize whole-head recording systems and Brain Electromagnetic Source Analysis (BESA; Scherg, 1990), a computer algorithm used to estimate the number and location of the neural generators producing scalp activity, have indicated that the ERN is generated by a single source in the medial frontal cortex (Dehaene, Posner, \& Tucker, 1994; Holroyd et al., 1998). A medial frontal source is consistent with Gehring et al.'s suggestion that the ERN is generated in the ACC. Although the neural generator of the Pe is less clear, Van Veen \& Carter (2002) have concluded that the Pe is also generated in the ACC.

In reviewing the functional role of the ACC, Bush, Luu, and Posner (2000) point out that there are at least two distinct regions of the ACC, as determined by cytoarchitecture, anatomical connectivity, and function: the "cognitive" dorsal/ventral ACC, and the "affective" rostral ACC. Consistent with this cognitive/ affective distinction, the ACC has been described by several researchers as part of a larger neural system that appears to integrate both affective and cognitive information. For instance, Devinsky, Morrell, and Vogt (1995) describe the ACC as part of the anterior executive region (AER) responsible for evaluating the motivational significance of stimuli within context-dependent situations, whereas Benaurroch $(1993,1997)$ positions the ACC within the central autonomic network (CAN) responsible for goal-directed actions and adaptability. Thayer and Lane (2000) propose that the CAN and AER are functionally identical, and that this single network "is associated with the process of response organization and selection, and serves to modulate psychophysiological resources in attention and emotion" (p. 205).

Such a modulatory role of the ACC on cardiovascular activity was demonstrated in a recent study by Critchley, Corfield, Chandler, Mathias, and Dolan (2000). Using positron emission tomography (PET) technology, they reported that changes in blood pressure were associated with the activation of the right ACC. Similar results have been reported for electrodermal activity (EDA). Fredrikson et al. (1998) found that EDA was positively related to bilateral activity in the ACC (see also Critchley, Elliot, Mathias, \& Dolan, 2000). Furthermore, Mangina and Beuzeron-Mangina (1996) report that intracerebral stimulation of the ACC results in EDA changes, and Tranel and Damasio (1994) report that lesions in the ACC are associated with blunted EDA.

As Bush et al. (2000) point out, there is currently no single theory that adequately accounts for all of the ACC experimental results. What is clear is that ACC activity is integrally involved in response appraisal - especially in conditions that require more cognitive control (MacDonald, Cohen, Stenger, \& Carter, 2000). Considering the ACC's involvement in response evaluation processes, its sensitivity to motivational and affective variables, and its influence on autonomic measures such as HR and EDA, it stands to reason that these ANS measures might also be sensitive to internal processes related to error detection.

In support of this possibility, Somsen, van der Molen, Jennings, and van Beek (2000) measured HR while adolescents performed the Wisconsin Card Sorting Test (WCST) and found a cardiac deceleration following negative feedback. In fact, the magnitude of this deceleration was sensitive to perseverative versus nonperseverative errors. Somsen et al. interpret these results as evidence for an error-related cardiac deceleration. A similar observation of cardiac deceleration following negative feedback has recently been reported by Crone et al. (in press), who also argue that error-related cardiac deceleration is associated with response monitoring.

If the ANS measures are functionally similar to the ERN, then it would be reasonable to assume that both heart rate deceleration and an increase in skin conductance would also occur when subjects detect their own errors in real time. The present study was conducted to investigate possible error-related ANS activity and its relationship to the ERN-Pe complex in a traditional error-monitoring procedure in which negative 
feedback was not provided. To this end, we recorded EEG, EKG, and SC activity while subjects performed a speeded reaction-time task that has been related to ACC activity in fMRI studies (Peterson et al., 1999). Specifically, we used a Stroop-type task in which subjects were presented with arrows pointing to the left or right hand and colored either red or green. The subjects' task was to respond to the color of the stimulus with a left or right key press as quickly and accurately as possible and to ignore the direction in which the arrow was pointing.

\section{Method}

\section{Participants}

Twenty-two undergraduate students at the University of Delaware (12 male, 10 female) served as subjects in the present experiment. For their participation, 9 students received credit as part of their introductory psychology course requirements and 13 students were paid $\$ 15$. The data from one participant was discarded due to near perfect task performance (one error). SCR data was lost from one participant due to experimenter error. No volunteers discontinued their participation in the experiment once informed consent was obtained and the procedures had begun.

\section{Task}

The modified Stroop task was administered on a Pentium I class computer, using Presentation software (Neurobehavioral Systems, Inc.) to control the presentation and timing of all stimuli, the determination of response accuracy, and the measurement of reaction times.

Throughout the task, participants were shown three large arrows oriented either to the right, to the left, or to the top of a 17-in. monitor. The arrows were positioned in the center of the screen and were presented either in red or green using a black background. A fixation mark (+) was presented just prior to the onset of each stimulus. The participants were instructed to press the left or right "ctrl" key with the left and right hands, respectively, in response to the color of the arrow and to disregard its orientation. In this way, the task contained congruent conditions (orientation of arrow pointing to correct hand), incongruent conditions (orientation of arrow pointing to incorrect hand), and neutral conditions (orientation of arrow irrelevant).

\section{Procedure}

After a brief description of the experiment, EEG/EOG, SCR, and HR sensors were attached and the participant was given detailed task instructions. Each participant was seated $0.5 \mathrm{~m}$ directly in front of the computer monitor and given two blocks of 18 practice trials. In one condition, the participants were told to press the left ctrl key when the arrow was red, and the right ctrl key when the arrow was green. In the other condition, the correspondence between the keys and arrow color was reversed. These conditions were counterbalanced across participants. The participants were told to place equal emphasis on speed and accuracy in their responses. Following practice, the participants received 12 blocks of 48 trials (576 total trials), with each block initiated by the participant. Arrow stimuli were presented for $200 \mathrm{~ms}$ at random intervals between 5,300 and 5,700 ms.
Psychophysiological Recording, Data Reduction, and Analysis

The electroencephalogram (EEG) was recorded using a Neurosoft Quik-Cap. Recordings were taken from three locations along the midline: frontal $(\mathrm{Fz})$, central $(\mathrm{Cz})$, and parietal $(\mathrm{Pz})$. In addition, Med-Associates miniature $\mathrm{Ag}-\mathrm{AgCl}$ electrodes were placed on the left and right mastoids (A1 and A2, respectively). During the recording, all activity was referenced to $\mathrm{Cz}$. The electrooculogram (EOG) generated from blinks and vertical eye movements was also recorded using Med-Associates miniature electrodes placed approximately $1 \mathrm{~cm}$ above and below the participant's right eye. The right earlobe served as a ground site. All EEG/EOG electrode impedances were below $10 \mathrm{~K} \Omega$ and the data from all channels were recorded by a Grass Model 7D polygraph with Grass Model $7 \mathrm{P} 1 \mathrm{~F}$ preamplifiers (bandpass $=0.05-35 \mathrm{~Hz}$ ).

Heart rate was obtained by attaching a Grass Photoelectric Transducer Model PPS to the participant's left ear lobe. The photocell output was fed into a Grass Model 7P1 Low Level DC Preamplifier and Model 7D Driver Amplifier (Bandpass= 1.6-3.0 Hz) and then into a series of Coulbourn logic modules that did threshold detection and shaping prior to the online computation of interbeat intervals.

Skin conductance responses were recorded using a Coulbourn Model S21-22 constant voltage $(0.5 \mathrm{~V})$ skin conductance coupler. Med Associates Standard $\left(0.5 \mathrm{~cm}^{2}\right) \mathrm{Ag} / \mathrm{AgCl}$ electrodes were placed on the thenar and hypothenar eminence of the palm with Johnson \& Johnson KY Jelly used as an electrolyte.

All bioelectric signals were digitized on a laboratory microcomputer using VPM software (Cook, 1999). The EEG was sampled at $200 \mathrm{~Hz}$. Data collection began 1,500 ms prior to stimulus presentation and continued for $5,000 \mathrm{~ms}$. Off-line, the EEG for each trial was corrected for vertical EOG artifacts using the method developed by Gratton, Coles, and Donchin (1983; Miller, Gratton, \& Yee, 1988) and then rereferenced to the average activity of the mastoid electrodes. Trials were rejected and not counted in subsequent analysis if there were excessive physiological artifacts or if the reaction time was shorter than 200 or longer than $800 \mathrm{~ms}$. Finally, the EEG for each trial was timelocked to its respective reaction time and averaged across trials to yield error- and correct-trial ERPs for each electrode site.

To quantify the ERN, each data point after response onset was subtracted from a baseline equal to the average activity in a 200-ms window prior to the response. The ERN was then defined as the most negative peak occurring in a window from 0 to $150 \mathrm{~ms}$ postresponse. Pe was defined as the most positive data point in the window between the ERN and $525 \mathrm{~ms}$ following response execution.

Interbeat intervals obtained from the photocell were converted to heart rate in beats per minute per real-time epoch $(250 \mathrm{~ms})$. When epochs contained portions of two beats, each rate was weighted according to the fraction of the epoch that it occupied (Graham, 1978). Heart-rate waveforms were then generated by deviating quarter-second averages during a 3.0-s postresponse epoch from the quarter-second average immediately preceding stimulus onset. Twelve $(3 \mathrm{~s})$ quarter-second averages, along with the onset point, constituted the heart-rate data that were then submitted to statistical analysis.

Skin conductance was sampled at $50 \mathrm{cps}$. Although the short poststimulus recording epoch did not allow for the development of a discrete SCR in most instances, the epoch was quantified by visually identifying activity that began with an onset latency greater than $0.5 \mathrm{~s}$ postresponse and measuring the difference, in 
$\mu \mathrm{S}$, between the identified SC onset point and the maximum SC value present in the 3.0-s postresponse window.

Because there has been some suggestion that uniformly fast reaction times can give rise to stimulus-related activity in the response-locked ERN, and because correct trials vastly outnumber incorrect trials, the ERN and the two autonomic measures were evaluated for errors and a subset of correct trials that were matched to error trials on the basis of reaction times. In addition to equating the number and speed (RT) of error and correct trials, this matching procedure allowed a comparison of post-error slowing with potential RT slowing after equally fast correct trials.

Finally, because Yeung, Botvinick, and Cohen (2003) argue that error-related brain activity might best be viewed in terms of the resolution of response conflict, the possible role of response conflict in modulating ANS measures was evaluated. To this end, additional analyses compared incongruent errors and RT-matched incongruent correct trials to congruent/neutral errors and RT-matched congruent/neutral trials. If ANS measures are sensitive to errors, as such, then there should be no effect of stimulus incongruency. The ERN, ANS, and performance measures were statistically evaluated using SPSS (Version 10.1) General Linear Model software with the Greenhouse-Geisser correction applied to the $p$ values of multiple $d f$ repeated measures comparisons.

\section{Results}

\section{Performance Measures}

Participants made relatively few errors during the procedure, achieving $94 \%$ correct on trials with valid EEG data. To investigate Stroop-related performance effects, the number of errors for each type of trial (congruent, incongruent, and neutral) was calculated. Repeated-measures ANOVA indicates a significant main effect for trial type, $F(2,40)=4.30, p<.05$. Post hoc LSD analyses (Sheshkin, 1997) indicate that subjects made significantly more errors on incongruent trials (16.42) than on either congruent (4.95) or neutral trials (9.0). Error rates on neutral and congruent trials did not differ.

To investigate the slowdown effect after errors, the average RT on trials following errors was compared to the average RT on trials following correct responses for each participant. Error trials were associated with significantly slower RTs on the subsequent trial [ $490 \mathrm{~ms}$ vs. $434.6 \mathrm{~ms} ; F(1,20)=36.9, p<.001]$, relative to all correct trials. However, because error trials are typically much faster than correct trials, this slowdown effect could be due to simple regression toward the mean. To rule out this possibility, the slowdown effect was also examined for errors versus a subset of correct trials that were RT-matched to error trials. Although there appeared to be some regression, slowdown after errors was still significantly greater than slowdown after equally fast correct trials [ $490 \mathrm{~ms}$ vs. $441 \mathrm{~ms} ; F(1,20)=23.47, p<.001]$.

In an effort to determine if post-error slowing was related to post-error performance, accuracy on post-error trials for each subject was compared to performance accuracy following RT-matched correct trials. Although performance was not significantly better after errors than after RT-matched correct trials $(91.1 \%$ vs. $92.6 \%$ correct; $F<1)$, post-error accuracy was significantly correlated with post-error RT slowdown, $r=.448$, $p<.05$. That is, subjects who had more slowing after errors had better performance on post-error trials.

\section{Psychophsyiological Measures-All Errors and RT-Matched Correct Trials}

Because each ERP is response locked between 300 and $500 \mathrm{~ms}$ after the presentation of the stimulus, it is possible that some of the response-locked ERP activity could nonetheless be stimulus driven (Gehring et al., 1993; Scheffers, Coles, Bernstein, Gehring, \& Donchin, 1996). If RTs on error trials are faster and more uniform than correct trials, these trials might contribute stimulus-related artifact to the ERN (although see Vidal, Burl, Bonnet, Grapperon, \& Hasbroucq, in press). Likewise, correct trials were much more numerous than error trials, raising the possibility that differences in the averages, particularly those involving ANS measurements, may be confounded by habituation processes. To avoid each of these potential problems, we examined psychophysiological data from a subset of the correct trials for each subject that were matched to error trials on the basis of RT. This way, correct and error trials were equated in terms of both RT and number of trials. The response-locked average ERP waveforms for all errors and RT-matched correct trials are presented in Figure 1.

As anticipated, a negative deflection associated with error trials began shortly after response execution and peaked approximately $55 \mathrm{~ms}$ later, primarily at the frontal recording site. For statistical analyses, the ERN was defined as the most negative peak occurring in a window from 0 to $150 \mathrm{~ms}$ postresponse, after response-locked data were deviated from a baseline equal to the average activity in a $200-\mathrm{ms}$ window prior to the response. A 2 (trial type) $\times 3$ (electrode site) analysis of variance with Greenhouse-Geisser corrected $p$ values confirmed that the ERN was significantly greater when subjects made errors, $F(1,20)=14.24 p<.01$. The interaction of trial and site was also significant, $F(2,40)=6.01, p<.01$, confirming that the difference in the ERN magnitude between correct and incorrect trials depicted in Figure 1 was largest at the Fz and $\mathrm{Cz}$ recording sites. Thus, our results are consistent with previously reported ERN morphology and topography. Pe was defined as the most positive data point in the baseline-corrected window between the ERN and $525 \mathrm{~ms}$ following response execution. Pe had a central scalp distribution, $F(2,40)=13.65, p<.001$, and was also significantly associated with errors, $F(1,20)=45.90, p<.001$.

Figure 2 illustrates both the heart rate (left) and skin conductance data (right) associated with error and correct trials. To evaluate heart-rate slowing, average HR for both error and RT-matched correct trials was subjected to a 2 (trial) $\times 11$ (time) repeated measures ANOVA with orthogonal polynomial contrasts used to evaluate the time factor. As suggested by Figure 2, the trend analysis revealed a significant main effect for trial, $F(1,20)=8.94, \quad p<.01$, with error trials prompting more deceleration than correct trials. This difference between error and correct trials was also reflected by significant linear, $F(1,20)=9.98, \quad p<.01$, and cubic, $F(1,20)=9.18, \quad p<.01$, Trial $\times$ Time interactions.

To evaluate SCR, we compared the magnitude of the SCR on error trials with the magnitude of the SCR on RT-matched correct trials. A paired-sample $t$ test confirmed that incorrect responses prompted a substantial SCR that was virtually absent when the response was correct, $t(19)=3.51, p<.01$.

\section{Relationship among Measures}

To examine the relationship among the physiological responses associated with errors and the relationship of these measures to performance, simple bivariate correlations were computed. 
$\mathrm{Fz}$

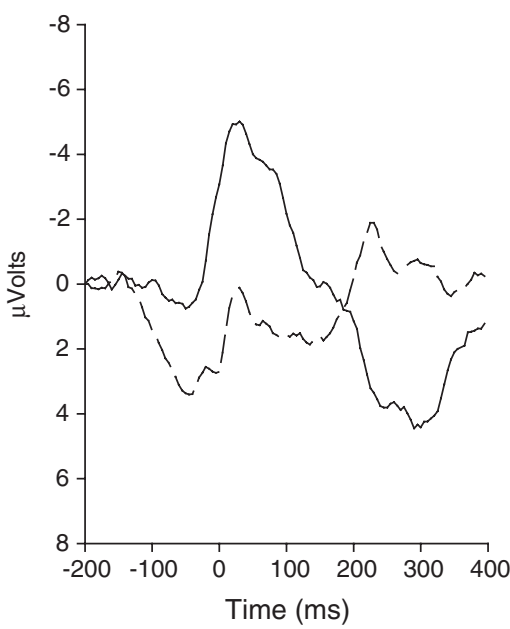

$\mathrm{Cz}$

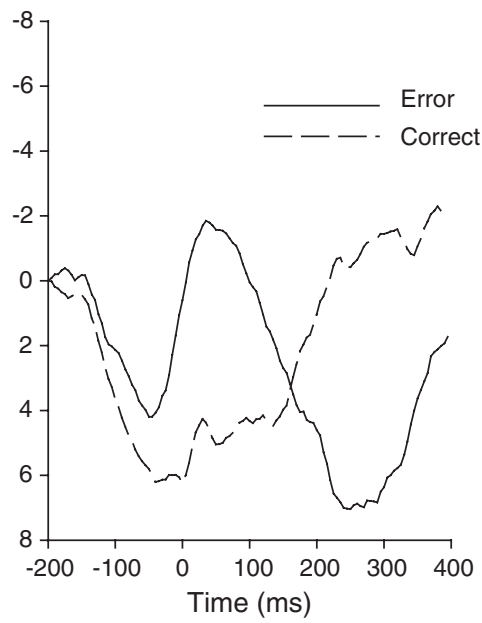

$\mathrm{Pz}$

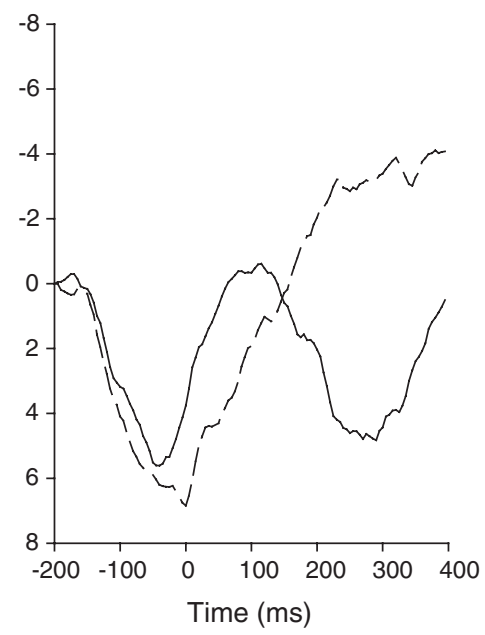

Figure 1. Averaged ERP waveforms from $\mathrm{Fz}, \mathrm{Cz}$, and $\mathrm{Pz}$ for all error trials and for an equal number of correct trials matched with error trials on response latency.
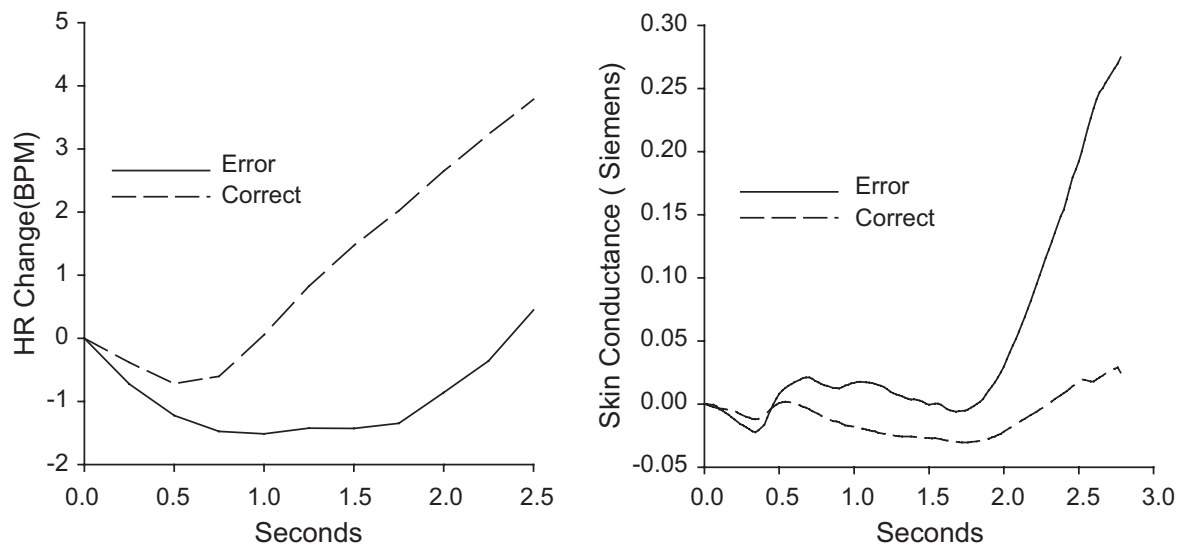

Figure 2. Averaged heart-rate (left) and skin-conductance-response (right) waveforms for all error trials and for an equal number of correct trials matched with error trials on response latency.

To control the Type I error rate, the number of measures included in the correlation matrix was severely culled. The results are presented in Table 1. The ERN did not correlate with Pe or with either HR deceleration or SCR magnitude. It did correlate with performance, however, with ERN magnitude decreasing as subjects made more errors. In contrast, there was no relationship between Pe and number of errors, but Pe was related to SCR and both the Pe and SCR were, in turn, related to post-error RT slowing.

ANS Measures-Incongruent versus Congruent/Neutral Errors Because subjects made more errors on incongruent trials, an explanation of the ANS activity in terms of error detection is confounded by stimulus congruency. That is, in terms of the

Table 1. Correlation Matrix of Primary Error-Related Measures

\begin{tabular}{|c|c|c|c|c|c|c|}
\hline & ERN (Fz) & $\mathrm{Pe}(\mathrm{Fz})$ & HR decel. & SCR $(\log )$ & \# errors & Slowdown \\
\hline $\begin{array}{l}\text { ERN (Fz) } \\
\text { Pe (Fz) } \\
\text { HR decel. } \\
\text { SCR (log) } \\
\text { \# errors }\end{array}$ & & .243 & $\begin{array}{r}.378 \\
-.143\end{array}$ & $\begin{array}{c}.053 \\
.548^{*} \\
-.290\end{array}$ & $\begin{array}{c}.584 * * \\
.055 \\
.394 \\
-.374\end{array}$ & $\begin{array}{c}-.051 \\
.479 * \\
-.294 \\
.571 * * \\
-.305\end{array}$ \\
\hline
\end{tabular}

$* p<.05 ; * * p<.01$. 
Table 2. Heart Rate and SCR during Incongruent and Congruent Conditions for Both Correct and Incorrect Responses

\begin{tabular}{lccccc}
\hline \hline & \multicolumn{2}{c}{ Heart rate $(\mathrm{BPM})$} & & \multicolumn{2}{c}{$\operatorname{SCR}(\mu \mathrm{S})$} \\
\cline { 2 - 3 } \cline { 5 - 6 } & Incongruent & Congruent & & Incongruent & Congruent \\
\hline Error & -0.64 & -0.65 & & 0.293 & 0.288 \\
Correct & 0.75 & 0.75 & & 0.042 & 0.125 \\
\hline \hline
\end{tabular}

response conflict model of error-related brain activity, it is possible that ANS measures were reactive to response conflict (incongruency), and not to error detection, as such. To test the possibility that ANS measures were sensitive to response conflict, we examined ANS data for incongruent error and RT-matched correct trials, and compared these data to congruent and neutral error and RT-matched correct trials. Because subjects made few congruent and neutral errors relative to incongruent errors, congruent and neutral trials were collapsed for purposes of the present analysis.

The ANS data for incongruent versus congruent/neutral trials are presented in Table 2 . For SCR, a 2 (trial type) $\times 2$ (congruency) repeated measures ANOVA confirmed the impression that although there was a main effect for errors, $F(1,19)=6.84, p<.05$, there was no effect for congruency, $F(1,19)<1$, and no Trial $\times$ Congruency interaction, $F(1,19)<1$. Similarly, a 2 (trial type) $\times 2$ (congruency) repeated measures ANOVA on the 2.5-s post-response HR yielded a significant effect for trial type, $F(1,20)=8.63, p<.01$, and again, there was no effect for congruency, $F(1,20)<1$, and no Trial $\times$ Congruency interaction, $F(1,20)<1$.

\section{Discussion}

\section{Performance Measures}

As in previous studies, we found that subjects tended to make more errors on incongruent trials. We also found that error trials were associated with increased reaction times on subsequent trials, even when post-error slowing was compared to the slowdown after equally fast correct trials. Although post-error slowing is construed as a compensatory mechanism geared toward improving performance on subsequent trials (Gehring \& Fencsik, 2001), we found that subjects did not perform more accurately after error trials than they did after RT-matched correct trials in general. The possibility that post-error slowing may be unrelated to compensatory behavior was raised by Gehring et al. (1993), who proposed that post-error slowing may instead be related to the same process that initially caused the execution error. In other words, Gehring et al. suggest that both the error and the post-error RT slowing could result from a single lapse in response monitoring. In the present context, however, this possibility is unlikely. Because of our use of relatively long intertrial intervals, post-error slowing occurred nearly $5 \mathrm{~s}$ after the commission of an error. This would imply an unrealistically long lapse in a single response monitoring process.

More importantly, we did find a significant positive correlation between post-error slowing and post-error accuracy. This would indicate that post-error RT slowing may well have been compensatory - at least under these experimental conditions. It is striking that even after a 5-s ITI, subjects still slow down following an error, and that the amount of this post-error slowing relates to the likelihood of success on the next trial. The fact that overall accuracy following errors does not increase may be due to near ceiling performance by most subjects. Recall that overall accuracy in the task was $94 \%$. Thus, despite the fact that subjects do not perform better after errors in general, the significant positive relationship between post-error performance and post-error RT slowing supports an interpretation of RT slowing as related to post-error compensatory processes.

\section{Psychophysiological Measures}

Consistent with previous studies, we found a frontocentrally maximal negative deflection in the event-related brain potential associated with the commission of errors (Dikman \& Allen, 2000; Falkenstein et al., 2000; Gehring et al., 1993; Luu, Collins, et al., 2000; Nieuwenhuis et al., 2001; Scheffers \& Coles, 2000). As in previous studies, this negative ERP component was followed by a central positivity, together comprising the typical ERN-Pe error complex (Falkenstein et al., 2000; Hohnsbein et al., 1989; Nieuwenhuis et al., 2001; Van Veen \& Carter, 2002).

Besides the two ERP components, we also found ANS activity that was specific to error trials. Thus, our original hypothesis that ANS activity might reflect endogenous response monitoring processes was confirmed. The association of significant cardiac deceleration with the commission of errors is consistent with Somsen et al.'s (2000) finding that negative feedback on the WCST prompted heart-rate slowing. The current results now extend the Somsen et al. findings regarding external feedback to error-related cardiac deceleration reflecting internal processes associated with response monitoring.

In addition to error-related bradycardia, we found that errors also gave rise to a substantial skin conductance response and that this error-related SCR activity was significantly correlated with the Pe. This pattern of results suggests that although the ERN and Pe share a close temporal relationship, the Pe and SCR might share a more functional similarity as psychophysiological indices of a response-monitoring system. This similarity is particularly evident when relationships with post-error compensatory slowing are examined - like the Pe, error-related SCR activity predicted the amount of post-error slowing.

Finally, we considered the possibility that ANS measures were sensitive to the degree of response conflict, rather than to error detection, per se. We found no indication that ANS measures were differentially sensitive to trials high or low in response conflict. Of course, our results do not address the validity of the conflict theory as it relates to the ERN. Rather, our analyses only indicate that conflict does not influence the response-monitoring processes indexed by ANS measures. In fact, Yeung et al. (2003) point out that the response monitoring system must, at some point, differentially process errors and correct responses. After all, people typically know when they have erred. Our data indicate that ANS measures may index these subsequent error-detection and compensatory processes.

\section{Relations between Psychophysiology and Behavior}

Existing ERN theories predict that the ERN should be related to post-error slowing (Botvinick, Braver, Barch, Carter, \& Cohen, 2001; Coles, Scheffers, \& Fournier, 1995). However, ERN magnitude has been related to this slowing only inconsistently, suggesting that the ERN may not be related to compensatory processes following errors (see Gehring et al., 1993, and Scheffers et al., 1996, for possible exceptions). In the present study also, no 
relationship between ERN magnitude and post-error slowing was observed. We did note, however, a significant inverse relationship between the size of the ERN and number of errors. This finding is consistent with a recent study of Pailing, Segalowitz, Dywan, and Davies (2002) in which they interpret the ERN as an index of response control-perhaps sensitive to impulsive responding. Although an interpretation of the relationship between errors and ERN amplitude based on response control is attractive, it is also possible that this relationship may reflect simple habituation to error commission rather than deficient response control. In fact, Holroyd and Coles (2002) argue that the ERN is a reinforcement learning signal, and as such, is sensitive to performance expectancy. Thus, the reinforcement learning theory of the ERN predicts that ERN magnitude and number of errors will be negatively correlated because poorer performance relates to lower expectations.

Accordingly, we found a relationship between ERN and number of errors but no relationship between ERN and posterror slowing. Interestingly, we found the opposite pattern of results for the error positivity: Pe was related to post-error slowing, but not to the number of errors. Specifically, error trials with larger Pe were associated with more post-error slowing. Although Nieuwenhuis et al. (2001) did not report a correlation between Pe amplitude and post-error slowing, they did find that unperceived errors were associated with both reduced $\mathrm{Pe}$ and a lack of post-error slowing. Thus, the finding in the present study of a relationship between Pe amplitude and post-error slowing is consistent with the Nieuwenhuis et al. data, and suggests that $\mathrm{Pe}$, and not ERN, is related to compensatory post-error RT slowing.

In fact, Nieuwenhuis et al. (2001) suggest that the Pe is a neural signal related to subsequent processes such as the awareness of errors and compensatory efforts. The present data suggest, going beyond Nieuwenhuis et al. (2001), that the awareness of error commission involves an additional critical component: visceral experience. In the present context, at least, it appears that when subjects make errors, they not only "know" that they erred, they "feel" it. In other words, the visceral experience associated with errors is indexed by error-related ANS activity and this error-related ANS activity is integrally involved in both the conscious awareness of having made a error and subsequent error compensation. Recall that, in fact, the errorrelated measure that correlated best with post-error compensatory slowing was SCR. This is reminiscent of Damasio's (1996) argument that emotion plays a crucial role in what otherwise appear to be seemingly rational processes.

In the error-processing literature, research typically focuses on events that occur on error trials themselves, and on events associated with trials subsequent to errors. This between-trial distinction essentially involves error-detection and error-com- pensation processes, respectively. In terms of this dichotomy, data from the present study suggest that the ERN is more related to error-detection processes, whereas the Pe and SCR are related to subsequent error awareness and compensation.

Although in the present experiment, subjects had the luxury of seconds to adjust their behavior following an error, the majority of ERN-type procedures involve much more closely spaced trials. From these studies, it is clear that compensatory behavior that follows an error takes place on the order of hundreds of milliseconds and cannot, therefore, be dependent on the perception of a fully developed ANS response. If the visceral reactivity associated with errors occurs after compensatory posterror slowing, why would these ANS measures correlate so strongly with post-error slowing?

Two possibilities suggest themselves. First, it may be that the ANS activity is not a precursor to compensatory behavior, but simply indicates that these compensatory processes have been put into place. In short, it is possible that the ANS activity reflects the end point of the sequence and does not play a role in the adjustment process itself. Alternatively, it may be, as Damasio (1996) suggests, that rational processes may utilize somatic information. For instance, prefrontal areas may actually make use of ANS information in action and response monitoring. This information, however, need not itself be present. Similar to what Damasio refers to as the "as-if" signal, it may be sufficient that the brain processes that generate the information that leads to ANS output supply, in parallel, an efferent copy to those processes that result in post-error behavioral compensation. Specifically, it may be that the Pe functions as this kind of dualpurpose "as-if" signal serving both to trigger subsequent errorrelated ANS activity and to signal frontal areas that behavioral compensation is required.

In sum, our data extends the growing literature on the interplay of emotional factors and psychophysiological indices of response monitoring. Importantly, both $\mathrm{HR}$ and SCR are sensitive to the commission of errors, extending previous response monitoring studies that have focused mainly on neural indices. Whereas previous studies have indicated affective modulation of the ERN, the current study demonstrates how Pe relates to the visceral response that follows an error. Importantly, both this ANS response and Pe appear related to post-error compensatory behavior, and we suggest that the Pe may actually serve two functions related to error awareness and subsequent compensation: The Pe may trigger error-related ANS activity in response-monitoring processes and act as a parallel signal to prefrontal areas, which presumably implement compensatory post-error behavior. In this way, the full range of response monitoring processes may rely on ERN, Pe, and ANS activity. Within this framework, ANS activity could be viewed as a "somatic marker" of erring (Damasio, 1996).

\section{REFERENCES}

Benarroch, E. E. (1993). The central autonomic network: Functional organization, dysfunction, and perspective. Mayo Clinic Proceedings, 68, 988-1001.

Benarroch, E. E. (1997). The central autonomic network. In P. A. Low (Ed.), Clincial Autonomic Disorders (2nd ed., pp. 17-24). Philadelphia: Lippincott-Raven.

Bernstein, P. S., Scheffers, M. K., \& Coles, M. G. H. (1995). "Where did I go wrong?" A psychophysiological analysis of error detection.
Journal of Experimental Psychology: Human Perception \& Performance, 21, 1312-1322.

Botvinick, M. M., Braver, T. S., Barch, D., Carter, C. S., \& Cohen, J. D. (2001). Conflict monitoring and cognitive control. Psychological Review, 108, 624-652.

Bush, G., Luu, P., \& Posner, M. I. (2000). Cognitive and emotional influences in anterior cingulate cortex. Trends in Cognitive Sciences, 4 , 215-222. 
Coles, M. G. H., Scheffers, M. K., \& Fournier, L. (1995). Where did you go wrong? Errors, partial errors, and the nature of human information processing. Acta Psychologica, 90, 129-144.

Cook, E. W., III (1999). VPM reference manual. Birmingham, Alabama: Author.

Critchley, H. D., Corfield, D. R., Chandler, M. P., Mathias, C. J., \& Dolan, R. J. (2000). Cerebral correlates of autonomic cardiovascular arousal: A functional neuroimaging investigation in humans. Journal of Physiology, 523, 259-270.

Critchley, H. D., Elliot, R., Mathias, C. J., \& Dolan, R. J. (2000). Neural activity relating to generation and representation of galvanic skin conductance responses: A functional magnetic resonance imaging study. The Journal of Neuroscience, 20, 3033-3040.

Crone, E., van der Veen, F., van der Molen, M., Somsen, R., van Beek, B., \& Jennings, R. (in press). Cardiac concomitants of feedback processing. Biological Psychology.

Damasio, A. R. (1996). The somatic marker hypothesis and the possible functions of the prefrontal cortex. Philosophical Transactions of the Royal Society of London Series B-Biological Sciences, 351, $1413-1420$.

Dehaene, S., Posner, M. I., \& Tucker, D. M. (1994). Localization of a neural system for error detection and compensation. Psychological Science, 5, 303-305.

Devinsky, O., Morrell, M. J., \& Vogt, B. A. (1995). Contributions of anterior cingulate cortex to behavior. Brain, 118, 279-306.

Dikman, Z. V., \& Allen, J. J. B. (2000). Error monitoring during reward and avoidance learning in high- and low-socialized individuals. Psychophysiology, 37, 43-54.

Falkenstein, M., Hoormann, J., Christ, S., \& Hohnsbein, J. (2000). ERP components on reaction errors and their functional significance: A tutorial. Biological Psychology, 51, 87-107.

Fredrikson, M., Furmark, T., Olsson, M. T., Fischer, H., Andersson, J., \& Langstrom, B. (1998). Functional neuroanatomical correlates of electrodermal activity: A positron emission tomographic study. Psychophysiology, 35, 179-185.

Gehring, W. J., Coles, M. G. H., Meyer, D. E., \& Donchin, E. (1990). The error-related negativity: an event-related brain potential accompanying errors. Psychophysiology, 27, S34.

Gehring, W. J., \& Fencsik, D. E. (2001). Functions of the medial frontal cortex in the processing of conflict and errors. The Journal of Neuroscience, 21, 9430-9437.

Gehring, W. J., Goss, B., Coles, M. G. H., Meyer, D. E., \& Donchin, E. (1993). A neural system for error detection and compensation. Psychological Science, 4, 385-390.

Gehring, W. J., Himle, J., \& Nisenson, L. G. (2000). Action-monitoring dysfunction in obsessive-compulsive disorder. Psychological Science, $11,1-6$.

Gehring, W. J., \& Knight, R. T. (2000). Prefrontal-cingulate interactions in action monitoring. Nature Neuroscience, 3, 516-520.

Gehring, W. J., \& Willoughby, A. R. (2002). The medial frontal cortex and the rapid processing of monetary gains and losses. Science, 295, 2279-2282.

Graham, F. K. (1978). Constraints on measuring heart rate and period sequentially through real and cardiac time. Psychophysiology, 15, 492-495.

Gratton, G., Coles, M. G. H., \& Donchin, E. (1983). A new method for off-line removal of ocular artifact. Electroencephalography and Clinical Neurophysiology, 55, 468-484.

Hajcak, G., McDonald, N., \& Simons, R. F. (in press). Anxiety and error-related brain activity. Biological Psychology.

Hajcak, G., \& Simons, R. F. (2002). Error-related brain activity in obsessive-compulsive undergraduates. Psychiatry Research, 110, 63-72.

Hohnsbein, J., Falkenstein, M., \& Hoormann, J. (1989). Error processing in visual and auditory choice reaction tasks. Journal of Psychophysiology, 3, 320 .

Holroyd, C. B., \& Coles, M. G. H. (2002). The neural basis of human error processing: Reinforcement learning, dopamine, and the error-related negativity. Psychological Review, 109, 679-709.

Holroyd, C. B., Dien, J., \& Coles, M. G. H. (1998). Error-related scalp potentials elicited by hand and foot movements: Evidence for an output-independent error-processing system in humans. Neuroscience Letters, 242, 65-68.
Johannes, S., Wieringa, B. M., Nager, W., Rada, D., Dengler, R., Emrich, H. M., Munte, T. F., \& Dietrich, D. E. (2001). Discrepant target detection and action monitoring in obsessive-compulsive disorder. Psychiatry Research: Neuroimaging, 108, 101-110.

Luu, P., Collins, P., \& Tucker, D. M. (2000). Mood, personality, and selfmonitoring: Negative affect and emotionality in relation to frontal lobe mechanisms of error monitoring. Journal of Experimental Psychology: General, 129, 43-60.

Luu, P., Flaisch, T., \& Tucker, D. M. (2000). Medial frontal cortex in action monitoring. The Journal of Neuroscience, 20, 464-469.

Luu, P., Tucker, D. M., Derryberry, D., Reed, M., \& Poulsen, C. (2003). Electrophysiological responses to errors and feedback in the process of action regulation. Psychological Science, 14, 47-53.

MacDonald, A. W., III, Cohen, J. D., Stenger, V. A., \& Carter, C. S. (2000). Dissociating the role of the dorsolateral prefrontal and anterior cingulate cortex in cognitive control. Science, 288 , $1835-1938$.

Mangina, C. A., \& Beuzeron-Mangina, J. H. (1996). Direct electrical stimuluation of specific human brain structures and bilateral electrodermal activity. International Journal of Psychophysiology, $22,1-8$.

Mathlaon, D. H., Fedor, M., Faustman, W. O., Gray, M., Askari, N., \& Ford, J. M. (2002). Response-monitoring dysfunction in schizophrenia: An event-related brain potential study. Journal of Abnormal Psychology, 111, 22-41.

Miller, G. A., Gratton, G., \& Yee, C. M. (1988). Generalized implementation of an eye movement correction procedure. Psychophysiology, 25, 241-243.

Miltner, W. H. R., Braun, C. H., \& Coles, M. G. H. (1997). Eventrelated brain potentials following incorrect feedback in a timeestimation task: Evidence for a "generic" neural sysytem for error detection. Journal of Cognitive Neuroscience, 9, 788-798.

Nieuwenhuis, S., Ridderinkhof, K. R., Blom, J., Band, G. P. H., \& Kok, A. (2001). Error-related brain potentials are differentially related to awareness of response errors: Evidence from an antisaccade task. Psychophysiology, 38, 752-760.

Pailing, P. E., Segalowitz, S. J., Dywan, J., \& Davies, P. L. (2002). Error negativity and response control. Psychophysiology, 39, 198-206.

Peterson, B. S., Skudlarski, P., Gatenby, J. C., Zhang, H., Anderson, A. W., \& Gore, J. C. (1999). An fMRI study of Stroop word-color interference: Evidence for cingulate subregions subserving multiple distributed attentional systems. Biological Psychiatry, 45, 1237-1258.

Rabbit, P. M. A. (1981). Sequential reactions. In D. Holding (Ed.), Human skills (pp. 153-175). New York: Wiley.

Scheffers, M. K., \& Coles, M. G. H. (2000). Performance monitoring in a confusing world: Error-related brain activity, judgments of response accuracy, and type of errors. Journal of Experimental Psychology: Human Perception and Performance, 26, 141-151.

Scheffers, M. K., Coles, M. G. H., Bernstein, P., Gehring, W. J., \& Donchin, E. (1996). Event-related brain potentials and error-related processing: An analysis of incorrect responses to go and no-go stimuli. Psychophysiology, 33, 42-53.

Scheffers, M. K., Humphrey, D. G., Stanny, R. R., Kramer, A. F., \& Coles, M. G. H. (1999). Error-related processing during a period of extended wakefulness. Psychophysiology, 36, 149-157.

Scherg (1990). Fundamentals of dipole source potential analysis. In F. Grandori, M. Hoke, \& G. L. Romani (Eds.), Auditory evoked magnetic fields and electric potentials (pp. 40-69). Basel, Switzerland: Karger.

Sheshkin, D. J. (1997). Handbook of parametric and nonparametric statistical procedures. New York: CRC Press, Inc.

Somsen, R. J. M., Van der Molen, M. W., Jennings, J. R., \& van Beek, B. (2000). Wisconsin card sorting in adolescents: Analysis of performance, response times and heart rate. Acta Psychologica, 104, 227-257.

Thayer, J. F., \& Lane, R. D. (2000). A model of neurovisceral integration in emotion regulation and dysregulation. Journal of Affective Disorders, 61, 201-216.

Tranel, D., \& Damasio, H. (1994). Neuroanatomical correlates of electrodermal skin conductance responses. Psychophysiology, 31, 427-438.

Van't Ent, D., \& Apkarian, P. (1999). Motoric response inhibition in finger movement and saccadic eye movement: A comparative study. Clinical Neurophysiology, 110, 1058-1072. 
Van Veen, V., \& Carter, C. S. (2002). The timing of action-monitoring processes in the anterior cingulate cortex. Journal of Cognitive Neuroscience, 14, 593-602.

Vidal, F., Burl, B., Bonnet, M., Grapperon, J., \& Hasbroucq, T. (in press). Error negativity on correct trials: A reexamination of available data. Biological Psychology.
Yeung, N., Botvinick, M. M., \& Cohen, J. D. (2003). The neural basis of error detection: Conflict monitoring and the error-related negativity. Manuscript submitted for publication.

(Received June 19, 2002; ACCEPTed May 2, 2003) 